\title{
IL-36 $\gamma$ promotes psoriasis-like features in keratinocytes in an imiquimod-induced murine model of psoriasis
}

\author{
Wen-Ming Wang, Chao Wu, Yi-Meng Gao, Hong-Zhong Jin
}

Department of Dermatology, State Key Laboratory of Complex Severe and Rare Diseases, Peking Union Medical College Hospital, Chinese Academy of Medical Science and Peking Union Medical College, Beijing, China

Submitted: 1 July 2021; Accepted: 29 December 2021

Online publication: 1 January 2021

Arch Med Sci

DOI: https://doi.org/10.5114/aoms/145396

Copyright @ 2021 Termedia \& Banach

\section{Abstract}

Introduction: Psoriasis is a recurrent, chronic inflammatory skin disease with a complex pathogenesis. The disease imposes a heavy burden on patients. Interleukin (IL)-36y belongs to the IL-36 family and is predominantly expressed by epithelial cells. IL-36 $\gamma$ is upregulated in psoriasis lesions. However, the effects of IL-36 $\gamma$ in keratinocytes remain unclear.

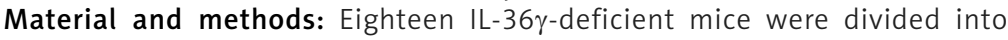
three groups: the Vaseline group, the imiquimod (IMQ) group, and the IMQ/ IL-36 $\gamma$ group. Vaseline or IMQ was administered for 6 consecutive days. The severity of psoriasis-like lesions was evaluated using a modified Psoriasis Area and Severity Index (PASI) scoring system. Production of cytokines and expression of differentiation markers were assessed by immunohistochemistry.

Results: IMQ-induced psoriasis lesions were significantly more severe in IMQ/IL-36 $\gamma$-treated mice compared with Vaseline-treated and IMQ-treated mice, as shown by an exacerbated inflammatory phenotype, increased numbers of blood vessels, increased infiltration of cells, and increased epidermal thickness. Expression of loricrin and keratin 5 in skin lesions was decreased following treatment with IL-36 $\gamma$. Levels of IL-17A, interferon- $\gamma, \beta$-catenin and Dickkopf-related protein 1 were elevated in keratinocytes within psoriatic

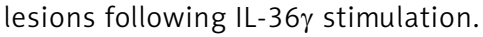

Conclusions: Together, these data showed that IL-36 $\gamma$ contributes to abnormal keratinocyte proliferation and keratinocyte-related proinflammatory cytokines, and suggest that IL-36 $\gamma$ may play an important role in the pathogenesis of psoriasis.

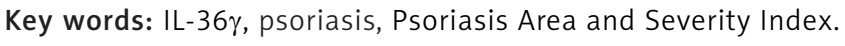

\section{Introduction}

Psoriasis is a chronic, immune-mediated inflammatory skin disorder that is characterized by well-demarcated, scaly, erythematous plaques. The mechanisms underlying psoriasis are not fully understood but the condition is generally attributed to dysfunction of the epithelial and immune systems triggered by stimuli such as infections, medications, and trauma. Psoriasis vulgaris is the most prevalent type of psoriasis, accounting for approximately $90 \%$ of all cases [1]. Histologically, psoriatic skin lesions are typically characterized by uncontrolled keratinocyte proliferation, aberrant differentiation of keratinocytes, dilated and

\author{
Corresponding author: \\ Hong-Zhong Jin MD \\ Department of \\ Dermatology \\ State Key Laboratory \\ of Complex Severe \\ and Rare Diseases \\ Peking Union Medical \\ College Hospital \\ Chinese Academy of \\ Medical Sciences and \\ Peking Union \\ Medical College \\ Shuaifuyuan Wangfujing \\ Dongcheng District \\ Beijing 100730 \\ China \\ Phone/fax: +86010 \\ 69151502 \\ E-mail: jinhongzhong@263.net
}


hyperplastic blood vessels, and inflammatory infiltration of leukocytes (including dendritic cells, macrophages, $T$ cells, and neutrophils) into the dermis [2]. The interactions among keratinocytes, cytokines, and immune cells play crucial roles in the formation of psoriatic lesion [2, 3].

Interleukin (IL)-36 cytokines, including IL-36 $\alpha$, IL-36 $\beta$ and IL-36 tokines. The IL-36 receptor antagonist (IL-36Ra) is an antagonist of IL-36 function [4]. IL-36 cytokines and IL-36Ra are abundant in the skin and participate in epidermal cornification. IL-36 is produced by various cell types including keratinocytes, epithelial cells, tissue resident macrophages, monocytes, and dendritic cells. Several studies have reported that expression of IL-36 $\gamma$ was upregulated in the lesions of psoriasis patients and was associated with disease activity $[5,6]$. However, the effect of IL-36y on keratinocytes remains unclear. In this study, we investigated the effects of IL-36y on keratinocytes in an imiquimod (IMQ)-induced murine model of psoriasis.

\section{Material and methods}

\section{Animal experiments}

All experimental mice were of the $(57 \mathrm{BL} / 6$ J background. IL-36 $\gamma$-deficient mice were generated by Shanghai Model Organisms (Shanghai, China). All mice were maintained under specific pathogen-free conditions and provided with a standard laboratory diet and water. The mice were randomly divided into three groups $(n=6$; three males and three females per group): the control group, the IMQ group, and the IMQ/IL-36 $\gamma$ group. Mice received a daily topical dose of $62.5 \mathrm{mg} 5 \%$ imiquimod cream (Aldara, 3M Pharmaceuticals, UK) or Vaseline on the shaved dorsal skin for 6 consecutive days. The mice in the IMQ/IL-36 $\gamma$ group

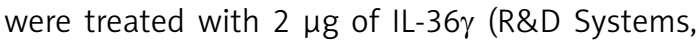
Minneapolis, MN, USA) by subcutaneous injection on days 1,3 , and 5 . The severity of psoriasis-like lesions was evaluated using a modified Psoriasis Area and Severity Index (PASI) scoring system. The modified system was adapted from the clinical PASI system, which includes three criteria: erythema, scaling, and thickness. Two parameters were scored independently on a scale from 0 to 4 according to external physical appearance: $0=$ none, 1 = slight, 2 = moderate, 3 = marked, and $4=$ severe.

\section{Immunohistochemistry}

Psoriatic skin samples from three groups were fixed in $10 \%$ formalin and embedded in paraffin for hematoxylin and eosin (H\&E) staining and histopathology. The sections were scanned using the Hamamatsu Digital Pathology System and epi- dermal thickness was measured using NDP.view software (Hamamatsu Photonics, Hamamatsu, Japan). For immunohistochemistry analysis, formalin-fixed and paraffin-embedded skin sections were incubated with primary antibodies against IL-17 (Abcam, Cambridge, MA, USA), IL-6 (Abcam), interferon (IFN)- $\gamma$ (Abcam), keratin 5 (Abcam), keratin 17 (Abcam), $\beta$-catenin (Abcam), and loricrin (Proteintech, Rosemont, IL, USA). Subsequently, the sections were incubated with horseradish peroxidase-conjugated secondary antibodies (Proteintech). Then, 3,3'-diaminobenzidine (Proteintech) was used as a substrate. The sections were scanned using the Hamamatsu Digital Pathology System.

The $\mathrm{H}$-score method was used to quantify staining intensity from immunohistochemistry. The range of the $\mathrm{H}$-score was $\mathbf{0 - 3 0 0}$ based on the percentage of cells at different staining intensities. The relative intensity of specific staining was defined as 0 (no staining), 1 (weak staining), 2 (moderate staining), and 3 (strong staining). The final score was the sum of the relative staining intensity (0-3) multiplied by the percentage of positive cells. The $\mathrm{H}$-score analysis was carried out using microscopy by two experienced pathologists in a double-blind fashion.

\section{Statistical analysis}

Data were presented as means \pm standard deviations. Statistical analysis was performed using SPSS 21.0 (IBM Corp., Armonk, NY, USA). Two-tailed tests were used in all analyses and values of $p<0.05$ were considered statistically significant. Differences in $\mathrm{H}$-score data and PASI scores were assessed using the Mann-Whitney $U$ test. Differences between continuous variables were assessed using an independent-sample $t$-test. Figures were produced using GraphPad Prism 8.0 (GraphPad Software Inc., San Diego, (A, USA).

\section{Results}

\section{IL-36 $\gamma$ aggravates the psoriasiform phenotype of IMQ-treated mice}

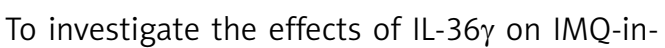
duced psoriasis in mice, IL-36 $\gamma$ was subcutaneously injected on days 1,3 , and 5 . The severity of psoriasiform lesions (thickening, scaling, and erythema) was evaluated using PASI scores. The skin of mice in the Vaseline group showed no psoriasis-like lesions (Figure 1 A). However, typical symptoms of scaling, thickness, and erythema were observed in the IMQ and IMQ/IL-36 $\gamma$ groups (Figures $1 \mathrm{~B}, \mathrm{C})$. Furthermore, the total PASI scores in the IMQ/IL-36 $\gamma$ group $(7.33 \pm 2.07$ vs. $9.83 \pm 1.33$, $p=0.015$ at day $5,6.42 \pm 1.63$ vs. $9.42 \pm 1.16$, 
A

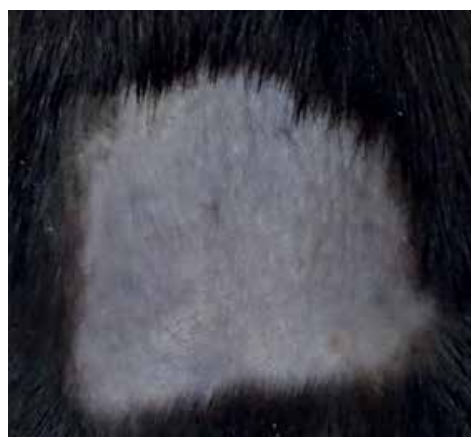

D

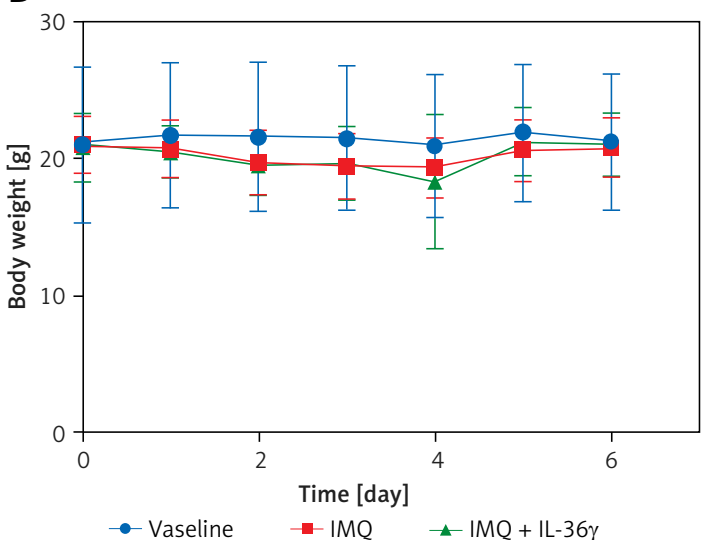

$\mathrm{F}$

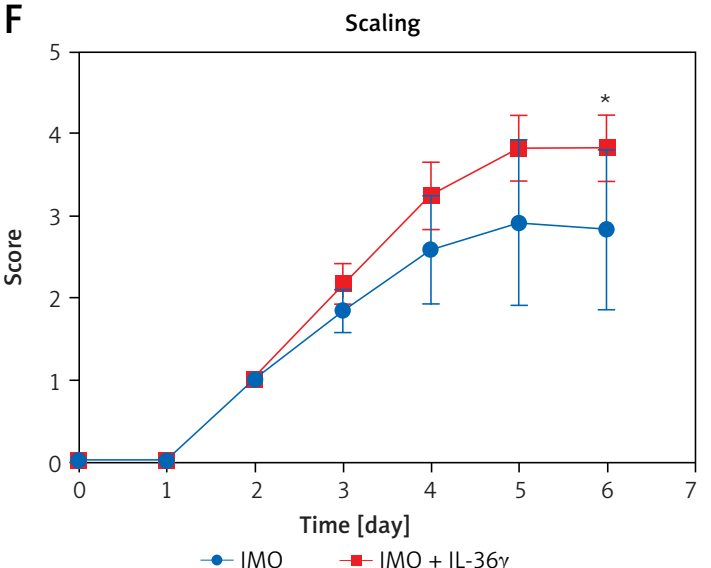

$\mathrm{H}$

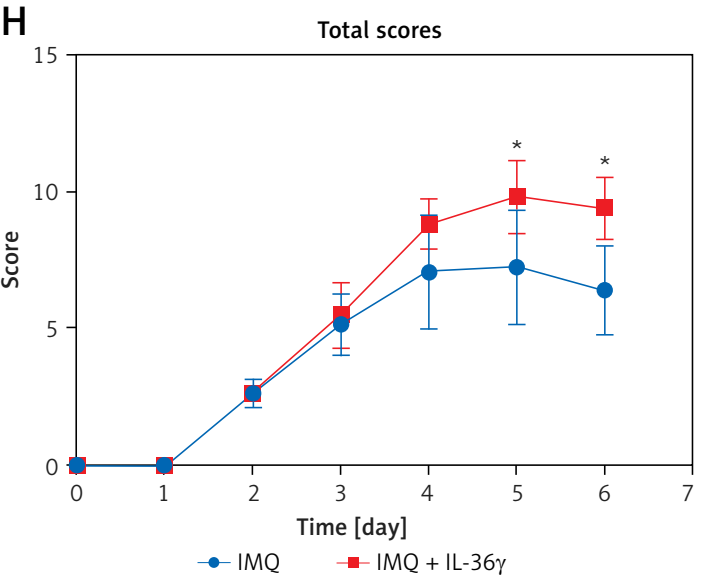

C IMQ + IL-36 $\quad$ IM

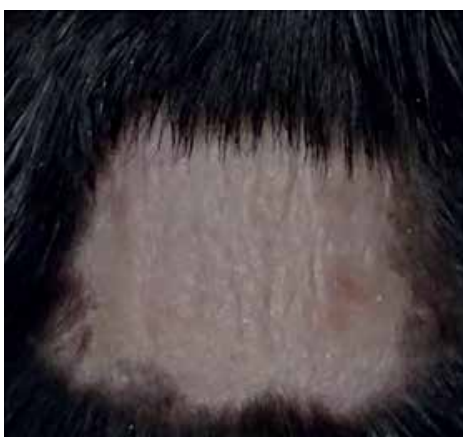

E

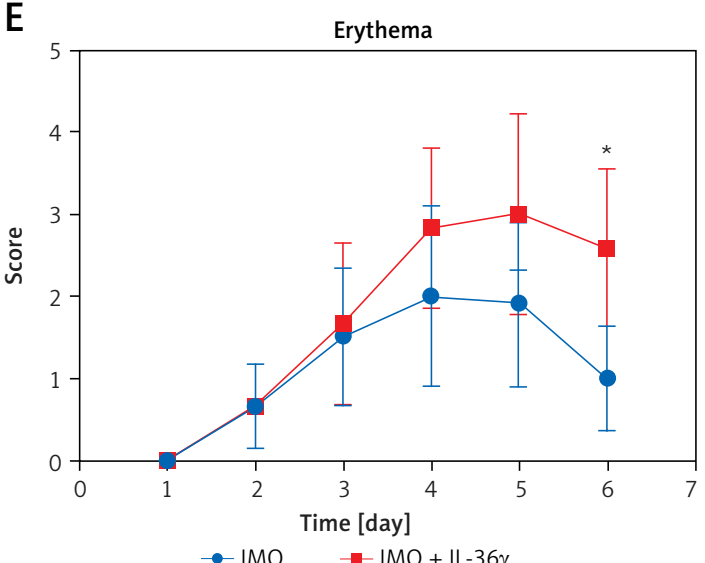

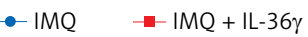

G

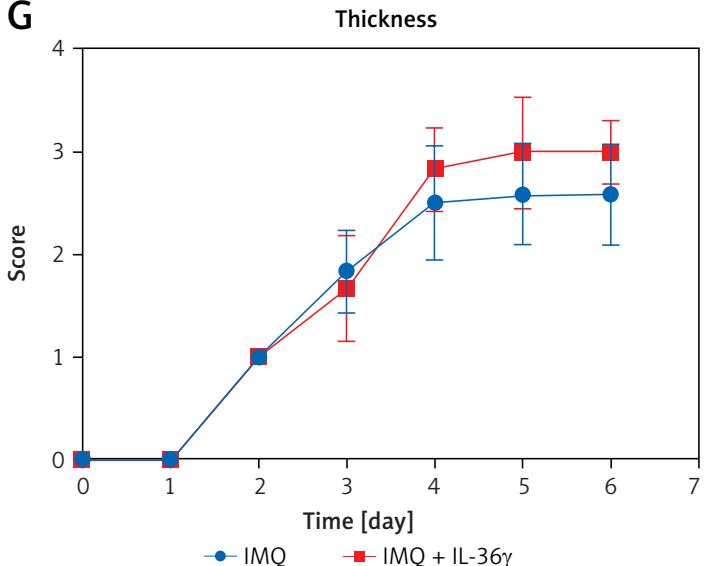

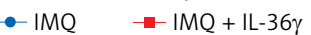

Figure 1. Effects of interleukin (IL)-36\% on morphological changes of imiquimod (IMQ)-induced psoriasiform lesions in mice. A-C - Representative pictures of the dorsal skin of mice from each group. D - Body weight change in each group from day 1 to day 6 . E-G - Severity of erythema (E), scaling $(\mathbf{F})$, and thickness $(\mathbf{G})$ was scored in each group daily. $\mathbf{H}$ - Cumulative scores (erythema + scaling + thickness) were calculated for each group daily. ${ }^{*} P<0.05$ 
$p=0.010$ at day 6) were higher than those in the IMQ group (Figures $1 \mathrm{E}-\mathrm{H}$ ). These results indicated that the clinical symptoms of psoriasiform lesions induced by IMQ treatment in mice can be significantly exacerbated by IL-36 $\gamma$.

\section{The histopathological characteristics} of psoriasiform lesions were aggravated in IL-36y-deficient mice following treatment

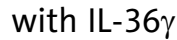

$H \& E$ staining was used to evaluate the histopathological characteristics of the dorsal lesions in mice of the three experimental groups (Vase-

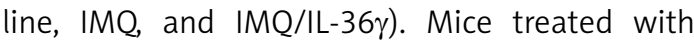

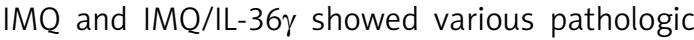
changes characteristic of psoriasis including epidermal hyperplasia, thickening of the acanthosis cell layer, inflammatory cell infiltration, and increased numbers of blood vessels (Figures $2 \mathrm{~A}-\mathrm{F}$ ).

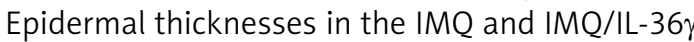
groups were $32.33 \pm 5.84$ and $47.83 \pm 3.25 \mu \mathrm{m}$, respectively, compared with only $22.98 \pm 5.94 \mu \mathrm{m}$ in the Vaseline group. Epidermal thickness in the IMQ/IL-36 $\gamma$ group was significantly higher than that in the IMQ group (Figure $2 \mathrm{G}$ ). Numbers of blood vessels and total cells in the dermis were

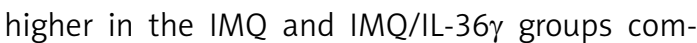
pared with the Vaseline group. Numbers of blood vessels and total cells in the dermis were higher in the IMQ/IL-36 $\gamma$ group compared with the IMQ group (Figures $2 \mathrm{H}, \mathrm{I}$ ).

\section{IL-36 $\gamma$ attenuates the expression of loricrin and keratin 5 in IL-36 $\gamma$-deficient mice}

To investigate the effects of IL-36y on keratinocyte differentiation, expression of loricrin, keratin 5 and keratin 17 in mouse skin lesions was assessed by immunohistochemistry. Semi-quantitative staining showed that loricrin expression was significantly lower in the IMQ and IMQ/IL-36r groups compared with the control group (Figures $3 \mathrm{~A}-\mathrm{D}$ ). Additionally, expression of loricrin and keratin 5 in skin lesions was decreased following treatment with IL-36y (Figures $3 \mathrm{E}-\mathrm{H}$ ). However, expression of keratin 17 was negative in the three groups of mice. These results demonstrated that IL-36 $\gamma$ treatment induced abnormal differentiation of keratinocytes following administration of IMQ (Figures $3 \mathrm{I}-\mathrm{K}$ ).

\section{IL-36 $\gamma$ induces psoriasis-associated IL-17A} and IFN- $\gamma$ expression in IL-36 $\gamma$-deficient mice

We next investigated the effects of IL-36y on expression of inflammatory mediators (IL-6, IL$17 \mathrm{~A}$, and IFN- $\gamma$ ) in the skin lesions of mice by immunohistochemistry.
IMQ exposure significantly increased the expression of IL-6, IL-17A, and IFN- $\gamma$. The markedly increased expression of IL-17A and IFN- $\gamma$, but not of IL-6, was further elevated following IL-36 $\gamma$ treat-

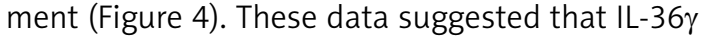
may exacerbate the inflammatory reactions of keratinocytes in a murine model of psoriasis.

Expression of $\beta$-catenin was upregulated in IL-36 $\gamma$-deficient mice following treatment

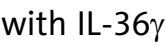

Expression levels of $\beta$-catenin and Dickkopf-related protein 1 (DKK1) in mice were assessed. As shown in Figure 5, $\beta$-catenin and DKK1 expression was elevated following IMQ stimulation (Figures $5 \mathrm{~A}, \mathrm{~B}, \mathrm{E}, \mathrm{F}$ ), and increased expression was en-

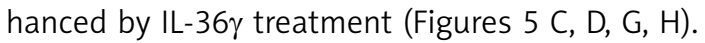
These results indicated that Wnt pathway signal-

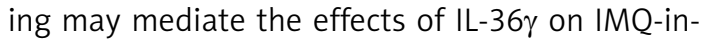
duced psoriasis.

\section{Discussion}

Psoriasis is a common, environmentally influenced, chronic inflammatory skin disorder affecting around $2-3 \%$ of the population. IL-36 is mainly restricted to keratinocytes and can contribute to skin inflammation by acting on keratinocytes and antigen-presenting cells [7, 8]. Previous studies showed that IL-36 $\gamma$ levels were significantly higher in lesions from psoriasis patients compared with healthy skin [8]. Using a murine model of IMQ-in-

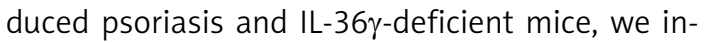

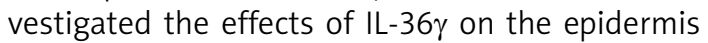
in psoriatic lesions. Our results demonstrated that IL-36 $\gamma$ exerted a positive effect on keratinocytes during the pathogenesis of psoriasis, promoting epidermal hyperplasia, inflammatory cell infiltration, increased vascularization, inhibition of differentiation, and pro-inflammation cytokine secretion.

The role of IL-36Ra in psoriasis has been previously studied. Treatment with IL-36Ra reduced the severity of IMQ-induced psoriasiform lesions in mice. BI 655130, a monoclonal antibody against the interleukin-36 receptor, has been used for the treatment of generalized pustular psoriasis [9]. Consistent with the results of these studies, we demonstrated that IL-36 $\gamma$ can exacerbate the clinical phenotype of IMQ-induced psoriatic dermati-

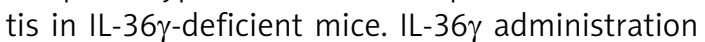
significantly increased the epidermal thickness of IMQ-induced psoriasiform lesions. These data suggested that IL-36 $\gamma$ may play an important role in the pathogenesis of psoriasis.

Although psoriasis is considered an immune-mediated disease, immune cells do not function in isolation. The interactions among im- 
A

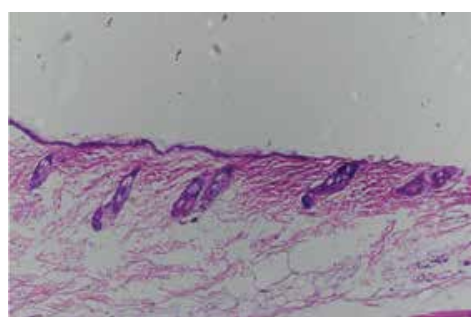

D

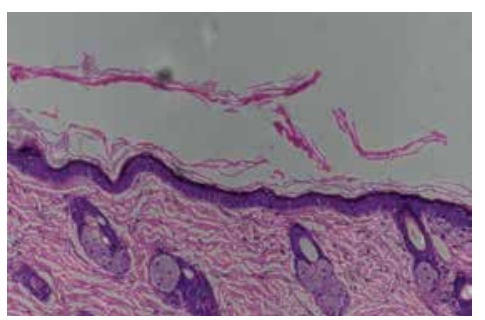

G

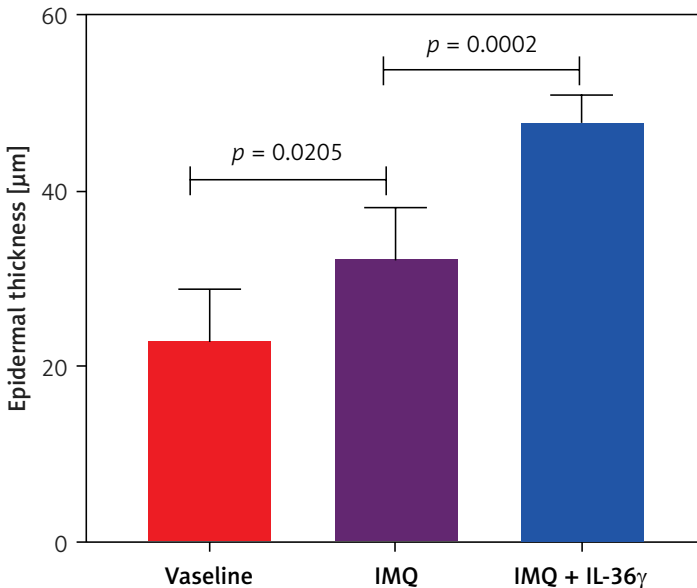

I

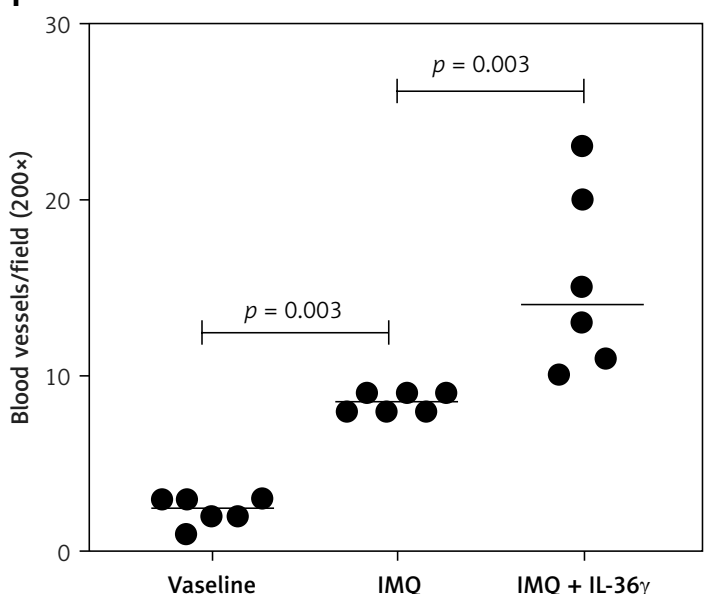

mune cells, keratinocytes, and cytokines are also involved in the pathogenesis of psoriasis. Keratinocytes are the main cells of the skin barrier. Skin keratinocytes undergo sequential physiological processes including proliferation, differentiation, and cell death. Each process is characterized by

E
IMQ
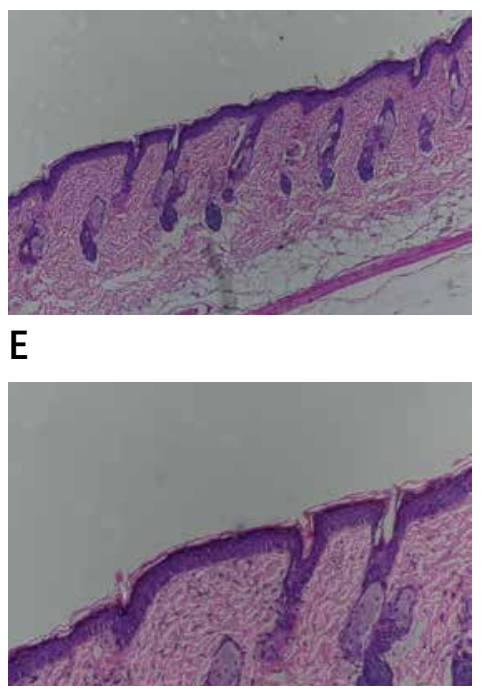

$\mathrm{H}$

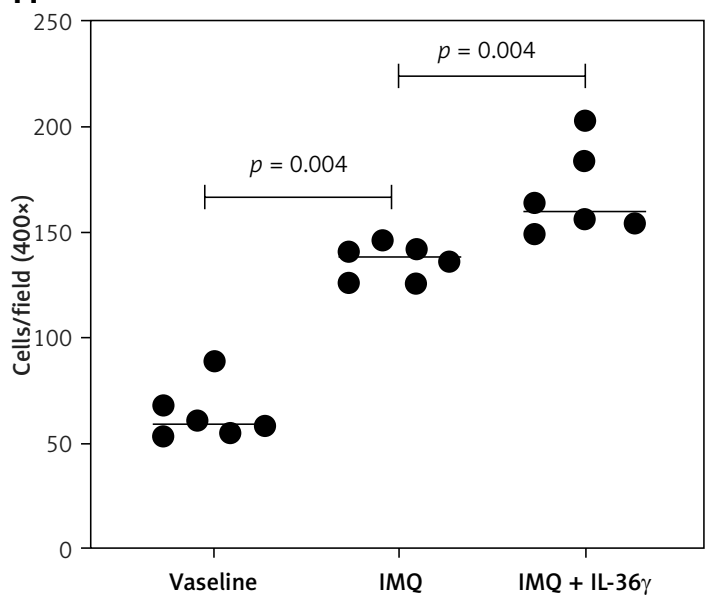

Figure 2. Effects of interleukin (IL)-36y on histological features of imiquimod (IMQ)-induced psoriasiform lesions in mice. A-C - Representative hematoxylin and eosin (H\&E) staining of the dorsal skin of mice in each group (magnification 100x). D-F - Representative H\&E staining of the dorsal skin of mice in each group (magnification 200x). G - Mean epidermal thickness in microns. $\mathbf{H}-$ Mean number of total cells in the dermis per field (magnification 400x). I - Mean number of blood vessels in the dermis per field (magnification 200x). ${ }^{\star} P<0.05$ 

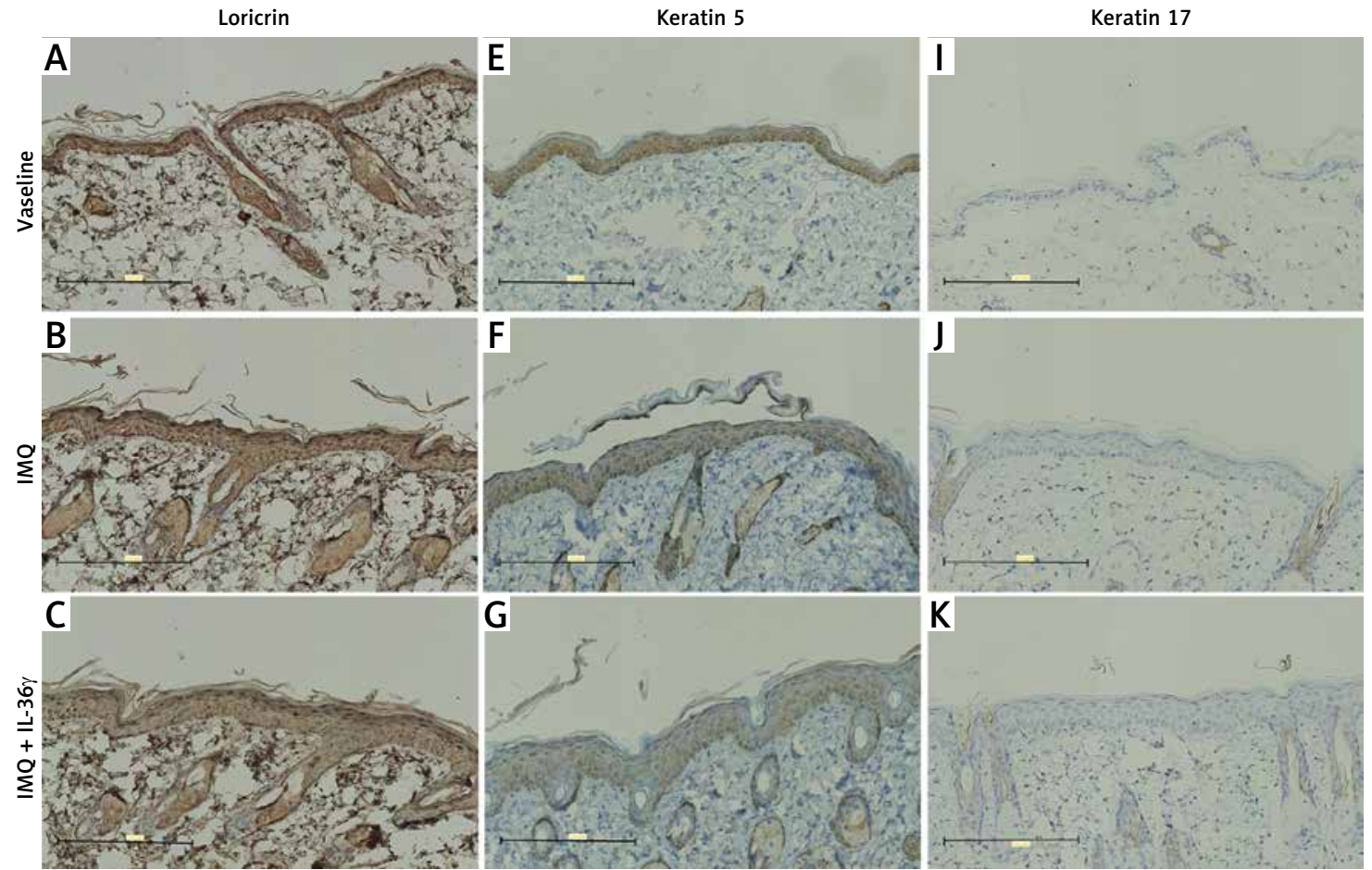

D

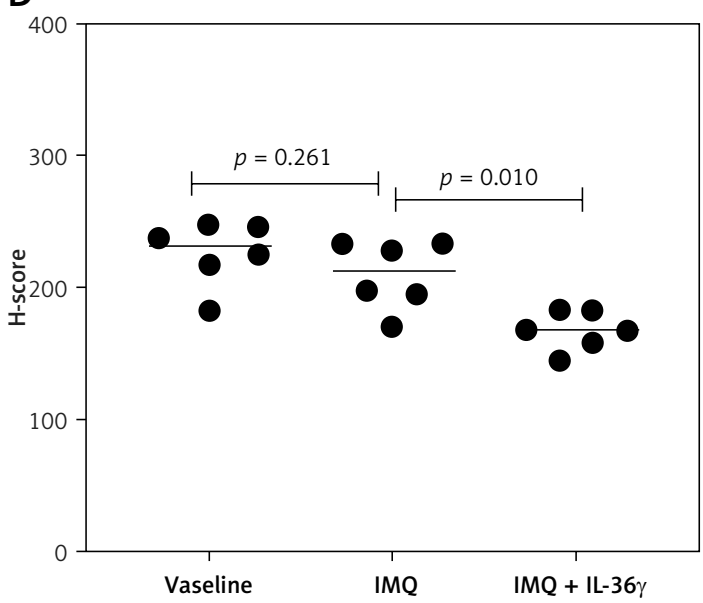

$\mathrm{H}$

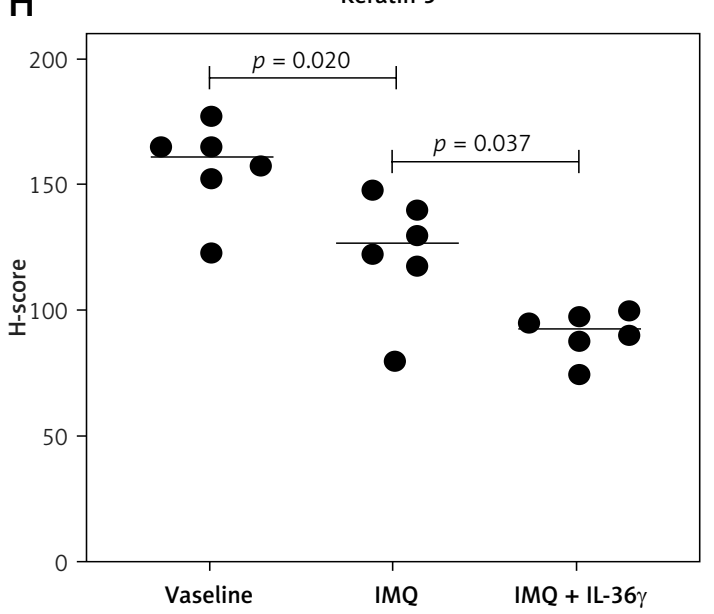

Figure 3. Interleukin (IL)-36y inhibited epidermal differentiation in an imiquimod (IMQ)-induced mouse model of psoriasis. Expression of loricrin $(\mathbf{A}-\mathbf{C})$, keratin $5(\mathbf{E}-\mathbf{G})$ and keratin $17(\mathbf{I}-\mathbf{K})$ was assessed in the epidermis of mice from each group (scale bars $200 \mu \mathrm{m}$ ). D, H - Mean $\mathrm{H}$-scores for loricrin and keratin 5 , respectively, in the epidermis of mice from each group. ${ }^{*} P<0.05$

Previous studies also showed that expression of loricrin was downregulated in the lesions of psoriasis patients and could be enhanced by treatment with tumor necrosis factor- $\alpha$ antagonists [11]. Keratin 5 is the main protein in basal keratinocytes [10]. Our study found that expression of loricrin and keratin 5 in psoriasis lesions was downregulated following IL-36 $\gamma$ treatment. Keratin 17 is an epithelial keratin and plays a role in the integrity of the epidermis. Keratin 17 is considered a marker of keratinocyte proliferation. After certain exogenous stressors, such as skin injury, keratin 17 can be induced in kerati- nocytes. Previous studies demonstrated that keratin 17 was expressed in the lesions of psoriasis patients and that its expression was positively associated with psoriasis severity. Furthermore, the expression of keratin 17 represents the highly activated and proliferative status of the epidermis in psoriasis [12]. These studies indicated that K17 may play an important role in the onset stage of psoriasis. $\mathrm{K} 17$ is considered an autoantigen for autoreactive T cells and the K17/T-cell/ cytokine autoimmune loop is implicated in the pathogenesis of psoriasis. However, a study also showed that K17 expression induced by IL-17A 

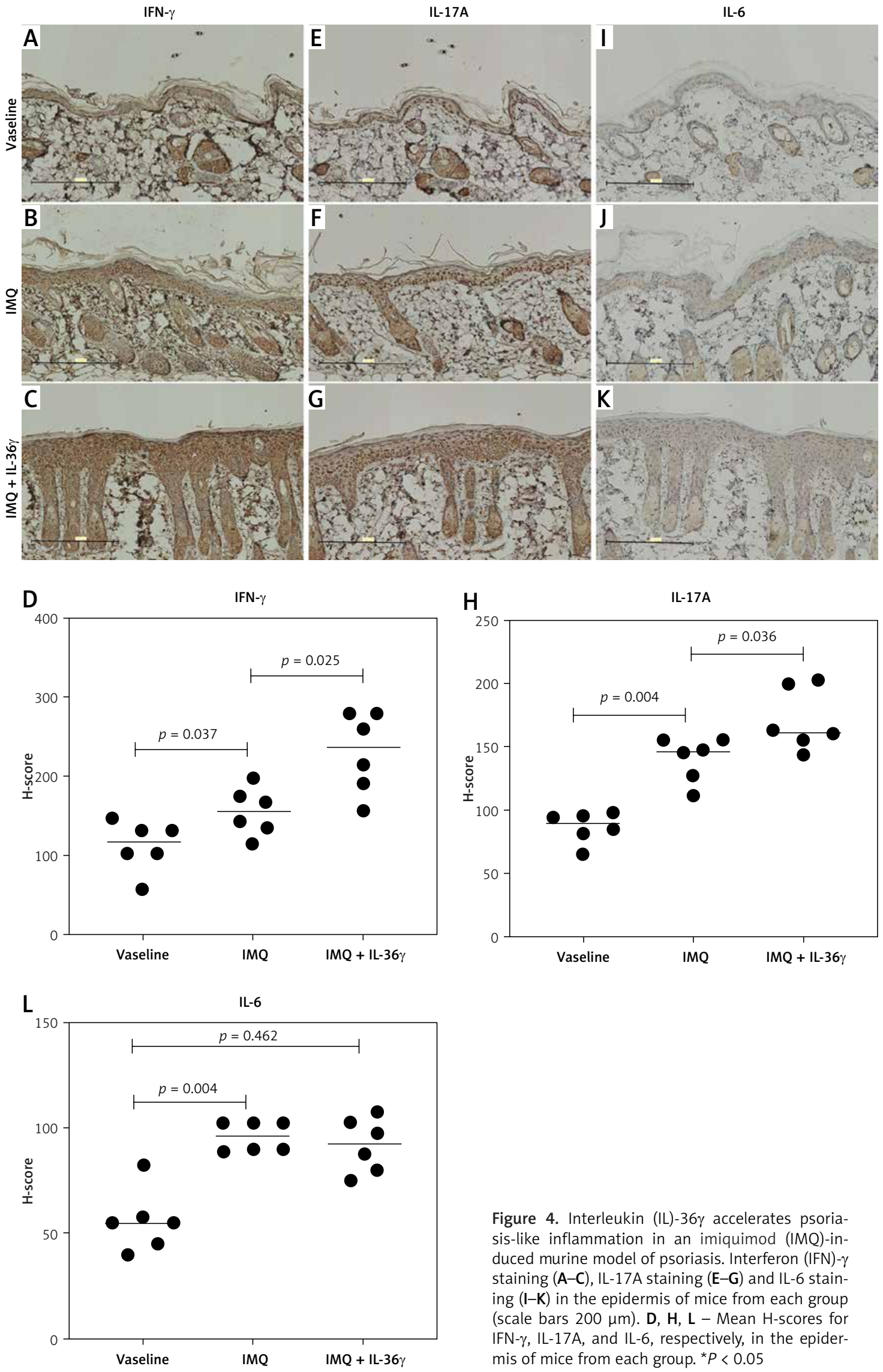

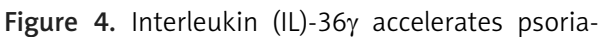
sis-like inflammation in an imiquimod (IMQ)-induced murine model of psoriasis. Interferon (IFN)- $\gamma$ staining (A-C), IL-17A staining (E-G) and IL-6 staining $(I-K)$ in the epidermis of mice from each group (scale bars $200 \mu \mathrm{m}$ ). D, H, L - Mean H-scores for IFN- $\gamma$, IL-17A, and IL-6, respectively, in the epidermis of mice from each group. ${ }^{*} P<0.05$ 

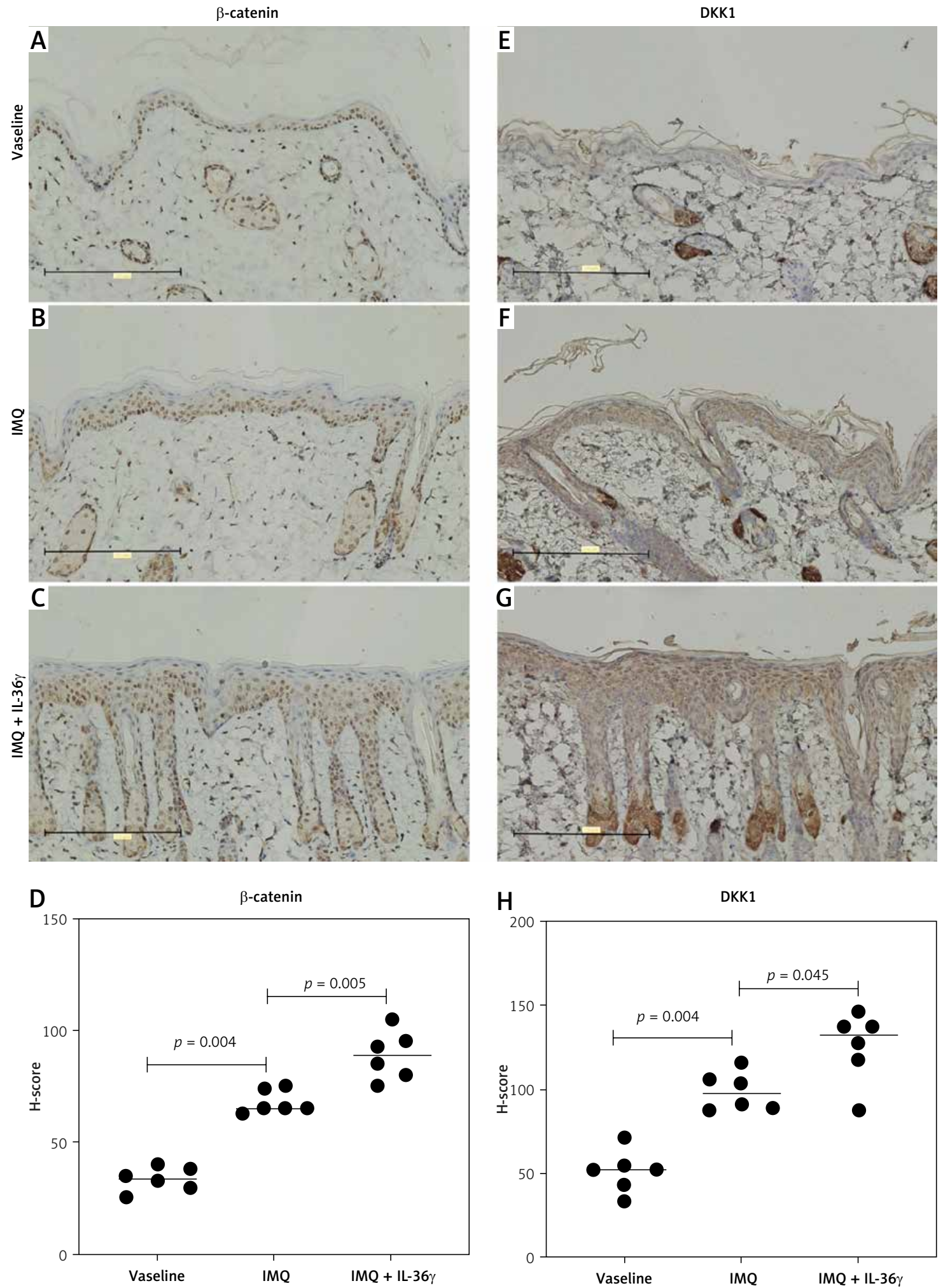

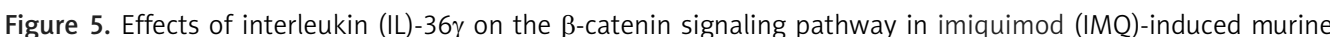
psoriasiform lesions. Expression of $\beta$-catenin $(\mathbf{A}-\mathbf{C})$ and DKK1 (E-G) was assessed in the epidermis of mice from each group (scale bars $200 \mu \mathrm{m}$ ). D, $\mathbf{H}$ - Mean H-scores for $\beta$-catenin and DKK1, respectively, in the epidermis of mice from each group. ${ }^{*} P<0.05$ 
in keratinocytes was dose-dependent [13]. In this study, we observed no expression of keratin 17 in any of the three groups of mice studied here. We speculate that expression of keratin 17 may be related to stage of psoriasis (e.g., the acute or chronic stage) and the psoriatic lesions of mice in our study were in the stable stage of psoriasis.

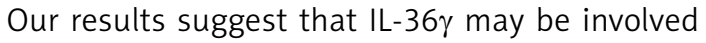
in the abnormal differentiation of keratinocytes and play a role in the pathogenesis of psoriasis.

Keratinocytes are the major constituents of the epidermis and act as the trigger and the executor in the pathogenesis of psoriasis. Keratinocytes are crucial in forming a physical barrier and are also major sources of inflammatory cytokines [14]. IL-17A is the main member of the IL-17 family and contributes to the pathogenesis of several diseases including psoriasis. Anti-IL-17A monoclonal antibodies, such as secukinumab and ixekizumab, can significantly alleviate psoriasis disease activity compared with placebo. IFN- $\gamma$ was recognized for its antiviral activity and is released by activated T cells and natural killer cells [15]. Previous work showed that in inflammatory settings, IFN- $\gamma$ could induce autocrine IFN- $\gamma$ production by keratinocytes [16]. Our results showed that the staining intensity of IL-17A and IFN- $\gamma$ in keratinocytes was

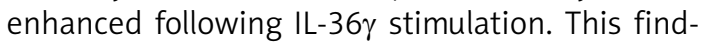
ing indicated that IL-36 $\gamma$ may be involved in innate immune system dysfunction in psoriasis. IL-6 can be expressed by human keratinocytes and promotes the proliferation of keratinocytes. Anti-IL- 6 therapies were effective for patients with psoriatic arthritis [17]. Our study demonstrated that the staining intensity of IL- 6 was not significantly different between the IMQ and IMQ/IL-36 $\gamma$ groups. In agreement with previous studies, our results suggested that IL-36 $\gamma$ may be involved in imbalanced innate and adaptive immunity in psoriasis.

$\beta$-catenin is a $94 \mathrm{kDa}$ protein that functions as a transcription factor of the Wnt signaling pathway. DKK-1 is an inhibitor of the canonical Wnt pathway [18]. Nuclear $\beta$-catenin expression was found to be enhanced in the lesions of psoriasis patients, while membranous $\beta$-catenin expression was lower in the lesions of psoriasis patients compared with controls [19]. However, another study showed the expression of $\beta$-catenin was lower in lesions from psoriasis patients compared with healthy controls [20]. Our study found that expression of $\beta$-catenin and DKK1 was elevated in an IMQ-induced murine model of psoriasis, and that increases in expression were enhanced by IL-36y treatment. We speculate that upregulation of DKK1 may represent feedback from the increase in $\beta$-catenin expression. Our findings indicate that $\beta$-catenin may mediate the role of IL-36 $\gamma$ in psoriasis, a possibility that will need to be clarified in further studies.
There are limitations of our study. First, our study was focused on the role of IL-36 $\gamma$ in an imiquimod-induced murine model of psoriasis and was not mainly designed to study the role of IL-36y in mice. Therefore, the mice treated only

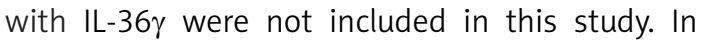
fact, adding the data of the mice treated only with

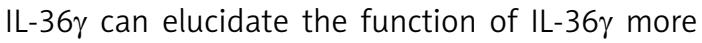
comprehensively. Second, our study describes a model of psoriasis in IL-36 $\gamma$-deficient mice, but the pathogenesis is not addressed in wild type mice. Future mechanistic work should address the role of IL-36y in wild type mice, which may facilitate elucidation of the mechanisms of IL-36y in the pathogenesis of psoriasis.

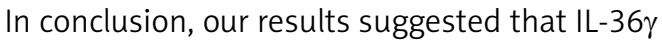
plays a role in keratinocyte activation in psoriasis. Uncontrolled keratinocyte proliferation, aberrant differentiation of keratinocytes, and inflammatory cytokine secretion were detected following IL$36 \gamma$ treatment in an IMQ-induced murine model

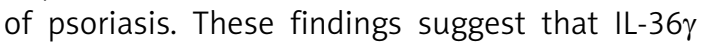
may represent a biomarker of psoriasis and suggest new directions toward development of antiIL-36R treatments for psoriasis patients. The role

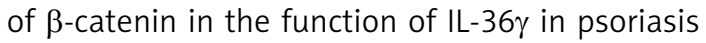
requires further study.

\section{Acknowledgments}

This study was supported by the National Natural Science Foundation of China (81773331, 82073450).

\section{Conflict of interest}

The authors declare no conflict of interest.

\section{References}

1. von Csiky-Sessoms S, Lebwohl M. What's new in psoriasis. Dermatol Clin 2019; 37: 129-36.

2. Deng Y, Chang C, Lu Q. The inflammatory response in psoriasis: a comprehensive review. Clin Rev Allergy Immunol 2016; 50: 377-89.

3. Hwang ST, Nijsten T, Elder JT. Recent highlights in psoriasis research. J Invest Dermatol 2017; 137: 550-6.

4. Neurath MF. IL-36 in chronic inflammation and cancer. Cytokine Growth Factor Rev 2020; 55: 70-9.

5. Ding L, Wang X, Hong X, et al. IL-36 cytokines in autoimmunity and inflammatory disease. Oncotarget 2018; 9: 2895-901.

6. Madonna S, Girolomoni G, Dinarello CA, et al. The significance of IL-36 hyperactivation and IL-36R targeting in psoriasis. Int J Mol Sci 2019; 20: 3318.

7. Mutamba S, Allison A, Mahida Y, et al. Expression of IL1 Rrp2 by human myelomonocytic cells is unique to $D C s$ and facilitates DC maturation by IL-1F8 and IL-1F9. Eur J Immunol 2012; 42: 607-17.

8. Balato A, Mattii M, Caiazzo G, et al. IL-36gamma is involved in. psoriasis and allergic contact dermatitis. J Invest Dermatol 2016; 136: 1520-3. 
9. Bachelez H, Choon SE, Marrakchi S, et al. Inhibition of the interleukin-36 Pathway for the treatment of generalized pustular psoriasis. N Engl J Med 2019; 380: 981-3.

10. Candi E, Schmidt R, Melino G. The cornified envelope: a model of cell death in the skin. Nat Rev Mol Cell Biol 2005; 6: 328-40.

11. Kim BE, Howell MD, Guttman-Yassky E, et al. TNF-alpha. downregulates filaggrin and loricrin through c-Jun $\mathrm{N}$-terminal kinase: role for TNF-alpha antagonists to improve skin barrier. J Invest Dermatol 2011; 131: 1272-9.

12. Lin Y, Zhang W, Li B, et al. Keratin 17 in psoriasis: current understanding and future perspectives. Semin Cell Dev Biol 2021; doi: 10.1016/j.semcdb.2021.06.018.

13. Moos S, Mohebiany AN, Waisman A, et al. Imiquimod-induced psoriasis in mice depends on the IL-17 signaling of keratinocytes. J Invest Dermatol 2019; 139: 1110-7.

14. Ni X, Lai Y. Keratinocyte: a trigger or an executor of psoriasis? J Leukoc Biol 2020; 108: 485-91.

15. Negishi H, Taniguchi T, Yanai H. The interferon (IFN) class of cytokines and the ifn regulatory factor (IRF) transcription factor family. Cold Spring Harb Perspect Biol 2018; 10: a028423.

16. Konur A, Schulz U, Eissner G, et al. Interferon (IFN)-gam$\mathrm{ma}$ is a main mediator of keratinocyte ( $\mathrm{HaCaT})$ apoptosis and contributes to autocrine IFN-gamma and tumour necrosis factor-alpha production. Br J Dermatol 2005; 152: 1134-42.

17. Mease PJ, Gottlieb AB, Berman A, et al. The efficacy and safety of. clazakizumab, an anti-interleukin-6 monoclonal antibody, in a phase iib study of adults with active psoriatic arthritis. Arthritis Rheumatol 2016; 68: 2163 73 .

18. Menet R, Bourassa P, Calon F, et al. Dickkopf-related protein-1 inhibition attenuates amyloid-beta pathology associated to Alzheimer's disease. Neurochem Int 2020; 141: 104881

19. El-Wahed Gaber MA, El-Halim Kandil MA, El-Farargy SM, et al. Beta-catenin expression in psoriasis. Indian Dermatol Online J 2015; 6: 13-6.

20. Farag AGA, Shoaib MA, Samaka RM, et al. Progranulin and. beta-catenin in psoriasis: an immunohistochemical study. J Cosmet Dermatol 2019; 18: 2019-26. 